\title{
Maximum standardized uptake value on FDG-PET predicts survival in stage I non-small cell lung cancer following carbon ion radiotherapy
}

\author{
KATSUYUKI SHIRAI ${ }^{1}$, TAKANORI ABE ${ }^{1}$, JUN-ICHI SAITOH $^{1}$, TATSUJI MIZUKAMI $^{1}$, \\ DAISUKE IRIE $^{1}$, YOSUKE TAKAKUSAGI ${ }^{1}$, SHINTARO SHIBA ${ }^{1}$, NAOKO OKANO ${ }^{1}$, \\ TAKESHI EBARA ${ }^{2}$, TATSUYA OHNO ${ }^{1}$ and TAKASHI NAKANO ${ }^{1}$ \\ ${ }^{1}$ Gunma University Heavy Ion Medical Center, Maebashi, Gunma 371-8511; \\ ${ }^{2}$ Department of Radiation Oncology, Gunma Prefectural Cancer Center, Ota, Gunma 373-8550, Japan
}

Received October 4, 2015; Accepted February 17, 2017

DOI: $10.3892 / 01.2017 .5952$

\begin{abstract}
The present study (University Hospital Medical Information Network study no. UMIN000003797) aimed to evaluate whether the maximum standardized uptake value $\left(\mathrm{SUV}_{\max }\right)$ of pretreatment ${ }^{18} \mathrm{~F}$-fluorodeoxyglucose-positron emission tomography (FDG-PET) is prognostic factor for stage I non-small cell lung cancer (NSCLC) treated with carbon ion radiotherapy (C-ion RT). Patients treated between June 2010 and June 2013 at Gunma University Heavy Ion Medical Center (Maebashi, Japan) on a prospective protocol were included in the present study. Patients with T1a-b and T2a NSCLC were treated with C-ion RT at a dose of $52.8 \mathrm{~Gy}$ [relative biological effectiveness (RBE)] and 60.0 Gy (RBE), respectively, in four fractions. Prior to treatment, all patients underwent FDG-PET, in which the $\mathrm{SUV}_{\max }$ of primary tumors was evaluated. Local control, progression-free survival (PFS), and overall survival (OS) were calculated. A total of 45 patients were analyzed and the median follow-up period was 28.9 months. The 2-year local control, PFS and OS rates for all patients were 93,78 and $89 \%$, respectively. The mean $\mathrm{SUV}_{\max }$ of primary tumors was 5.5 , and patients were divided into higher $(\geq 5.5)$ and lower $(<5.5) \mathrm{SUV}_{\max }$ groups. The 2-year PFS rates were 61 and $89 \%$ for the higher and lower $S U V_{\text {max }}$ groups, respectively $(\mathrm{P}=0.01)$, and the 2-year $\mathrm{OS}$ rates for the higher and lower $\mathrm{SUV}_{\max }$ groups were 76 and $96 \%$, respectively $(\mathrm{P}=0.01)$. The higher $\mathrm{SUV}_{\max }$ group exhibited a significantly worse PFS and OS compared with the lower $\mathrm{SUV}_{\max }$ group;
\end{abstract}

Correspondence to: Dr Katsuyuki Shirai, Gunma University Heavy Ion Medical Center, 3-39-22 Showa-machi, Maebashi, Gunma 371-8511, Japan

E-mail: kshirai@gunma-u.ac.jp

Key words: carbon ion radiotherapy, stage I non-small cell lung cancer, ${ }^{18} \mathrm{~F}$-fluorodeoxyglucose-positron emission tomography, maximum standardized uptake value, prognostic factor however, the $\mathrm{SUV}_{\max }$ was not associated with the local control rate. In total, 2 patients (4\%) experienced grade 2 or 3 radiation pneumonitis, with their symptoms improved through conservative treatment. No patients experienced any grade 4 or 5 toxicities. The results of the present study indicate that pretreatment $\mathrm{SUV}_{\max }$ is a prognostic indicator for outcomes in patients with stage I NSCLC treated with C-ion RT.

\section{Introduction}

In Japan, lung cancer is the leading cause of cancer-associated mortality (1). Although surgical resection is the typical treatment for stage I non-small cell lung cancer (NSCLC), the number of medically inoperable cases treated with radiotherapy has increased (2). Furthermore, patients with operable NSCLC may preferentially select radiotherapy in order to avoid surgery. Radiotherapy, including stereotactic body radiotherapy (SBRT) and particle beam therapy, serves an important role in the treatment of stage I NSCLC $(3,4)$.

Carbon ion radiotherapy (C-ion $\mathrm{RT}$ ) provides an improved dose distribution by the distal fall-off of the Bragg peak and less lateral scatter compared with photon therapy. These physical characteristics contribute to the delivery of high-dose radiation to the tumor, while minimizing the dose to surrounding normal tissue $(5,6)$. Furthermore, C-ion RT exhibits a larger mean linear energy transfer compared with other radiotherapy beams, which causes a high rate of cancer cell death due to the formation of double-stranded DNA breaks (7). Considering these advantages, C-ion RT is expected to achieve high local control rates without severe adverse events in several types of tumor. For stage I NSCLC, a number of studies of $\mathrm{C}$-ion $\mathrm{RT}$ revealed that its efficacy and safety were comparable to SBRT (8-10). Despite the high rate of local control in C-ion RT for stage I NSCLC, regional lymph node or distant metastasis are relatively common $(8,9)$. Identifying patients with a high risk of metastasis is important and adjuvant chemotherapy may decrease the level of metastasis in these patients. Therefore, the establishment of a pretreatment prognostic factor for recurrence and survival in NSCLC is required. 
${ }^{18}$ F-fluorodeoxyglucose-positron emission tomography (FDG-PET) has been revealed to be a useful technique for tumor staging through its ability to detect of tumor extension or metastasis (11-14). In several types of tumor, FDG-PET has been demonstrated to predict survival and treatment response (15-17). A meta-analysis of 13 studies on NSCLC treated with surgery revealed that a high FDG-PET standardized uptake value (SUV) of the primary tumor was a prognostic factor for unfavorable survival (18). However, the prognostic value of FDG-PET for stage I NLCLC treated with radiotherapy remains unclear. The aim of the present study was to evaluate whether the maximum SUV $\left(\mathrm{SUV}_{\max }\right)$ of pretreatment FDG-PET may predict the prognosis of stage I NSCLC treated with C-ion RT.

\section{Materials and methods}

Study design and patients. Since 2010, patients with peripheral stage I (T1a-2a N0 M0) NSCLC have been treated with C-ion RT at Gunma University Heavy Ion Medical Center (Maebashi, Japan). The present study analyzed the patients that were treated between June 2010 and June 2013 with a prospective protocol approved by the Institutional Review Board of Gunma University Hospital (Maebashi, Japan). The present study was registered at the University Hospital Medical Information Network Center (http://www.umin.ac.jp; study no. UMIN000003797). Tumor stage was determined through chest computed tomography (CT) scanning, brain magnetic resonance imaging and PET/CT within 1 month prior to $\mathrm{C}$-ion RT. Although the majority of patients exhibited biopsy-proven NSCLC, several patients avoided biopsy due to medical conditions. These patients were clinically diagnosed by subsequent tumor growth on CT scans and/or by accumulation on FDG-PET. Treatment options were based on discussions of the Cancer Board of the hospital, including surgical and medical oncologists, and a radiologist. All patients provided written informed consent prior to treatment.

Treatment planning. C-ion beams of 290, 380 and $400 \mathrm{MeV}$ were generated by the heavy particle accelerator at Gunma University Heavy Ion Medical Center. Details of the techniques for $\mathrm{C}$-ion $\mathrm{RT}$ and treatment planning have been previously described (19). CT simulation was performed following immobilization of the patients on fixation cushions and thermoplastic shells. Gross tumor volume was delineated as a visible lesion on lung window $\mathrm{CT}$ images. The clinical target volume margin was set at 5-8 $\mathrm{mm}$ to include subclinical disease extension. The planning target volume margin included set-up and internal margins, which were determined by tumor motion on four-dimensional CT images. The dose of C-ion RT was expressed as Gy [relative biological effectiveness (RBE)], which is defined as the physical C-ion dose $x$ RBE (3). Patients with T1a-b and T2a were treated with 52.8 Gy (RBE) and 60.0 Gy (RBE), respectively. All treatments were administered in four fractions within a week.

PET scan acquisition. All patients underwent FDG-PET prior to C-ion RT. Subsequent to fasting for $6 \mathrm{~h}$, FDG-PET scans were performed $60 \mathrm{~min}$ following intravenous FDG injection with a maximum activity of $400 \mathrm{Mbq}$. The $\mathrm{SUV}_{\max }$ of the primary tumor was determined by drawing the volume of interest on attenuation-corrected FDG-PET reconstructed images. The $\mathrm{SUV}_{\max }$ was automatically calculated based on the maximum activity in the volume of interest, injected FDG dose and patient weight (GE Healthcare Life Sciences, Chalfont, UK; Discovery ST Elite).

Assessment and follow-up. Patient follow-up included a clinical examination and CT scan every 3 months for the first year, and every 6 months subsequently. Local recurrence was defined as progressive abnormalities on CT images with accumulation on FDG-PET. The majority of recurrent cases of NSCLC were pathologically confirmed by biopsy, cytology or salvage surgery. Toxicities, including pneumonitis, dermatitis, esophagitis and chest wall pain, were scored according to the Common Terminology Criteria for Adverse Events version $4.0(20)$.

Statistical analysis. Local control, progression-free survival (PFS), and overall survival (OS) were measured using the Kaplan-Meier estimator method. The statistical significance of differences in local control and survival was assessed using the log-rank test. Local control, PFS and OS were calculated from the start date of C-ion RT until the last available follow-up or until the events, including recurrence, metastasis and death. Differences between two groups were compared using a Student's t-test. All statistical analyses were performed using SPSS software (version 21.0; SPSS Inc., Armonk, NY, USA). $\mathrm{P}<0.05$ was considered to indicate a statistically significant difference.

\section{Results}

Patient and tumor characteristics. A total of 45 patients were analyzed in the present study and their clinicopathological characteristics are presented in Table I. The median patient age was 72 years (range, 47-85). There were 18 patients (40\%) with T1a, 15 (33\%) with T1b and 12 (27\%) with T2a. Squamous cell carcinoma was diagnosed in 16 patients (36\%), adenocarcinoma in $24(53 \%)$ and clinically diagnosed lung cancer in $5(11 \%)$. The number of patients with operable NSCL was $30(67 \%)$ and the number with inoperable NSCLC was $15(33 \%)$. The median follow-up time for all patients was 28.9 months (range, 7.9-56.7). In total, 2 patients (4\%) experienced grade 2 or 3 radiation pneumonitis; however, their symptoms rapidly improved subsequent to conservative treatments. No patients experienced any grade 4 or 5 toxicities.

Local control, progression-free survival and overall survival. Fig. 1 shows the curves of local control, PFS and OS for the entire cohort. The 2-year local control rate, PFS and OS were 93,78 and $89 \%$, respectively. Of the 10 patients with recurrent NSCLC, 3 exhibited local recurrences, 2 exhibited hilar lymph node metastasis and 5 exhibited distant metastasis (data not shown). At the time of analysis, 8 patients had succumbed to lung cancer $(n=4)$ or comorbidities $(n=4)$ (data not shown).

Outcomes according to the $S U V_{\text {max }}$ of FDG-PET. The mean $\mathrm{SUV}_{\max }$ of primary tumors was 5.5 (range, 0.8-22.4), which was used as the cut-off to divide the cohort into two groups. 
Table I. Patient and tumor characteristics $(n=45)$.

\begin{tabular}{|c|c|c|}
\hline Characteristic & No. of patients & Frequency $(\%)$ \\
\hline \multicolumn{3}{|l|}{ Gender } \\
\hline Male & 31 & 69 \\
\hline Female & 14 & 31 \\
\hline \multicolumn{3}{|l|}{ ECOG performance status } \\
\hline 0 & 19 & 42 \\
\hline 1 & 24 & 53 \\
\hline 2 & 2 & 4 \\
\hline \multicolumn{3}{|l|}{ Operability } \\
\hline Operable & 30 & 67 \\
\hline Inoperable & 15 & 33 \\
\hline \multicolumn{3}{|l|}{ Histology } \\
\hline Squamous cell carcinoma & 16 & 36 \\
\hline Adenocarcinoma & 24 & 53 \\
\hline Clinical lung cancer & 5 & 11 \\
\hline \multicolumn{3}{|l|}{ T stage } \\
\hline T1a & 18 & 40 \\
\hline $\mathrm{T} 1 \mathrm{~b}$ & 15 & 33 \\
\hline $\mathrm{T} 2 \mathrm{a}$ & 12 & 27 \\
\hline \multicolumn{3}{|l|}{ RT dose } \\
\hline 52.8 Gy (RBE) & 33 & 73 \\
\hline 60.0 Gy (RBE) & 12 & 27 \\
\hline \multicolumn{3}{|l|}{ FDG-PET } \\
\hline $\mathrm{SUV}_{\max }<5.5$ & 29 & 64 \\
\hline $\mathrm{SUV}_{\max } \geq 5.5$ & 16 & 36 \\
\hline
\end{tabular}

ECOG, Eastern Cooperative Oncology Group; T, tumor; RT, radiotherapy; RBE, relative biological effectiveness; FDG-PET, ${ }^{18} \mathrm{~F}$-fluorodeoxyglucose-positron emission tomography; $\mathrm{SUV}_{\max }$, maximum standardized uptake value.

All patients were divided into a higher $(\geq 5.5)$ or lower $(<5.5) \mathrm{SUV}_{\max }$ group. Patient and tumor characteristics were not significantly associated with $\mathrm{SUV}_{\max }$ groups. The 2-year local control rates for the higher and lower $\mathrm{SUV}_{\max }$ groups were 87 and $96 \%$, respectively, which was not significantly different ( $\mathrm{P}=0.27$; Fig. 2). Patient clinicopathological characteristics were not significantly associated with the local control rate (data not shown). The 2-year PFS rates for the higher and lower $\mathrm{SUV}_{\max }$ groups were 61 and $89 \%$, respectively (Fig. 3). The higher $\mathrm{SUV}_{\max }$ group exhibited a significantly worse PFS compared with the lower $\mathrm{SUV}_{\max }$ group $(\mathrm{P}=0.01$; Fig. 3$)$. In addition, operability $(\mathrm{P}<0.01)$ and tumor stage $(\mathrm{P}=0.03)$ were significant prognostic factors for PFS (data not shown). The 2-year OS rates for the higher and lower $\mathrm{SUV}_{\max }$ groups were 76 and $96 \%$, respectively, and the difference betweem the two groups was statistically significant $(\mathrm{P}=0.01$, Fig. 4). Operability $(\mathrm{P}<0.01)$ and performance status $(\mathrm{P}=0.03)$ were also significant prognostic factors for OS (data not shown). Histological type was not significant factor for PFS and OS. Multivariate analysis was not performed due to the small number of patients in the present study.

\section{Discussion}

Based on a 2-year follow-up period, the present study revealed that C-ion RT produced 93\% local control and $89 \%$ OS for stage I NSCLC with minimal toxicity. Furthermore, a higher $\mathrm{SUV}_{\max }$ of the primary tumors on FDG-PET was significantly associated with a worse PFS and OS compared with a lower $\mathrm{SUV}_{\max }$. To the best of our knowledge, this is the first report demonstrating that pretreatment $\mathrm{SUV}_{\max }$ on FDG-PET is a prognostic factor for stage I NSCLC treated with C-ion RT.

In SBRT, the utility of $\mathrm{SUV}_{\max }$ as a prognostic factor for stage I NSCLC remains unclear; several previous studies have identified no significant association between $\mathrm{SUV}_{\max }$ and patient outcomes (21-22). Burdick et al (22) analyzed pretreatment $\mathrm{SUV}_{\max }$ in 72 patients with inoperable T1-2N0M0 NSCLC treated with SBRT and concluded that pretreatment $\mathrm{SUV}_{\max }$ did not predict for local control, mediastinal failure, distant metastases or OS. In Japan, Takeda et al (23) revealed that a high SUV $_{\max }$ of the primary tumor was associated with a significantly worse local control rate in 95 patients with NSCLC treated with SBRT. It was concluded that NSCLC tumors with a high $\mathrm{SUV}_{\max }$ may require dose escalation to improve local control, but patient outcomes were not studied. Chang et al (24) evaluated 130 patients with stage I NSCLC who underwent SBRT with $50 \mathrm{~Gy}$ in four fractions and concluded that a higher $\mathrm{SUV}_{\max }$ was markedly associated with worse PFS and OS, but not local control. In a study by Clarke et al (25) on 82 patients with inoperable stage I NSCLC treated with SBRT, it was concluded that pretreatment $\mathrm{SUV}_{\max }$ was associated with local relapse and distant metastases.

A previous meta-analysis of 13 studies on NSCLC treated with radiotherapy revealed that a high pre-treatment $\mathrm{SUV}_{\max }$ was significantly associated with unfavorable local control in patient treated with SBRT (hazard ratio, 1.11; 95\% confidence interval, 1.06-1.18) (26). In the present study, the higher $\mathrm{SUV}_{\max }$ group did not exhibit worse local control compared with the lower $\mathrm{SUV}_{\max }$ group, possibly as the local control rate of C-ion RT was high and the difference between two groups was not significant. Regardless of FDG accumulation, C-ion RT exhibits an advantage in the local control of primary tumor.

Several surgical studies have evaluated the prognostic value of pretreatment FDG-PET in NSCLC. Goodgame et al (27) revealed that a pretreatment $\mathrm{SUV}_{\text {max }} \geq 5.5$ predicted worse recurrence and survival in 136 patients treated with surgery, and suggested that stage I NSCLC with a high $\mathrm{SUV}_{\max }$ should be considered for adjuvant chemotherapy. In addition, Nair et al (28) systematically reviewed the association between FDG uptake and prognosis in stage I NSCLC treated with surgery. It was concluded that an increased tumor FDG uptake was associated with worse patient survival and could potentially be used as a biomarker for identifying high-risk patients with stage I NSCLC. Similarly, the results of the present study demonstrated that a high FDG accumulation was associated with worse PFS and OS.

A high FDG uptake suggests that metabolically active cancer cells take up glucose. Vesselle et al (29) reported that FDG uptake is associated with tumor proliferation and 


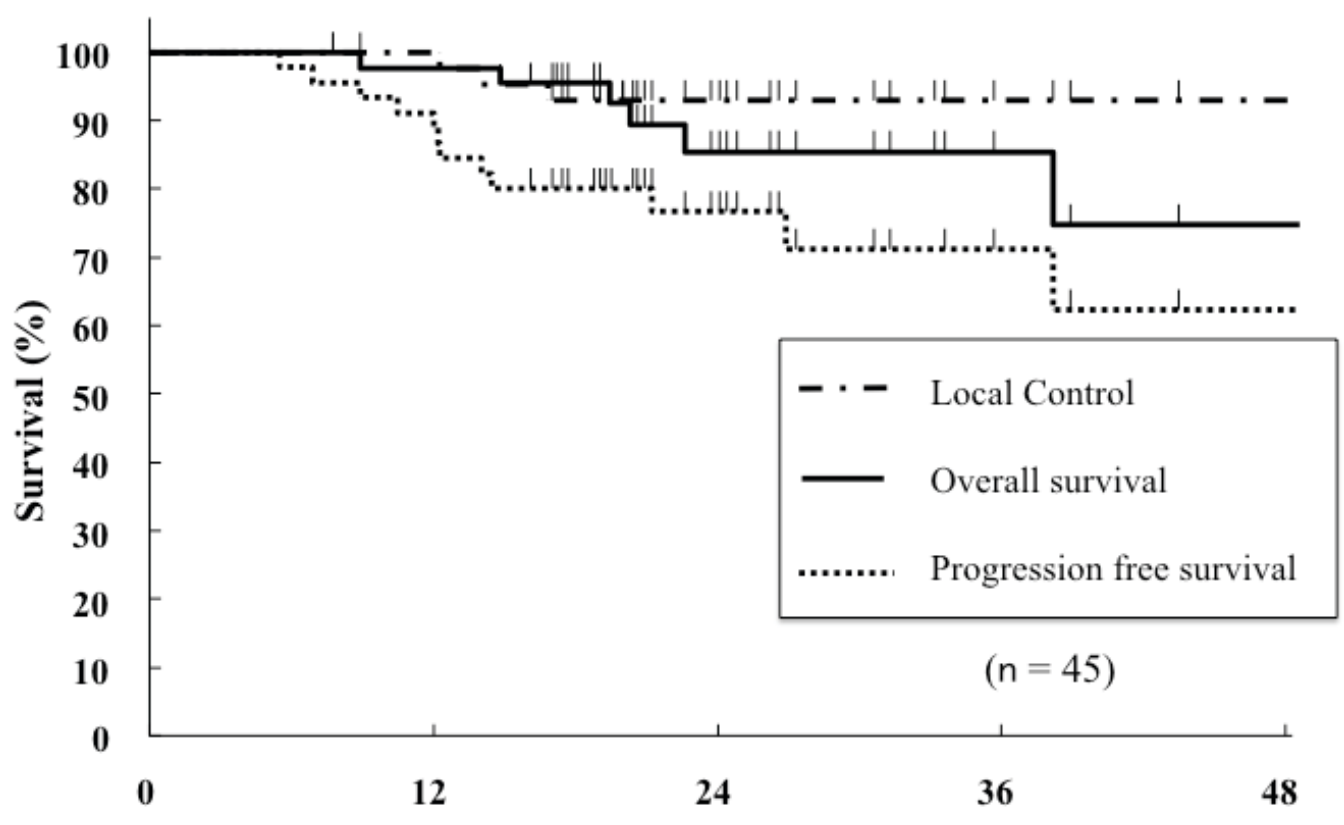

\section{Months}

Figure 1. Local control, overall survival and progression-free survival curves for patients with stage I non-small cell lung cancer following treatment with carbon ion radiotherapy.

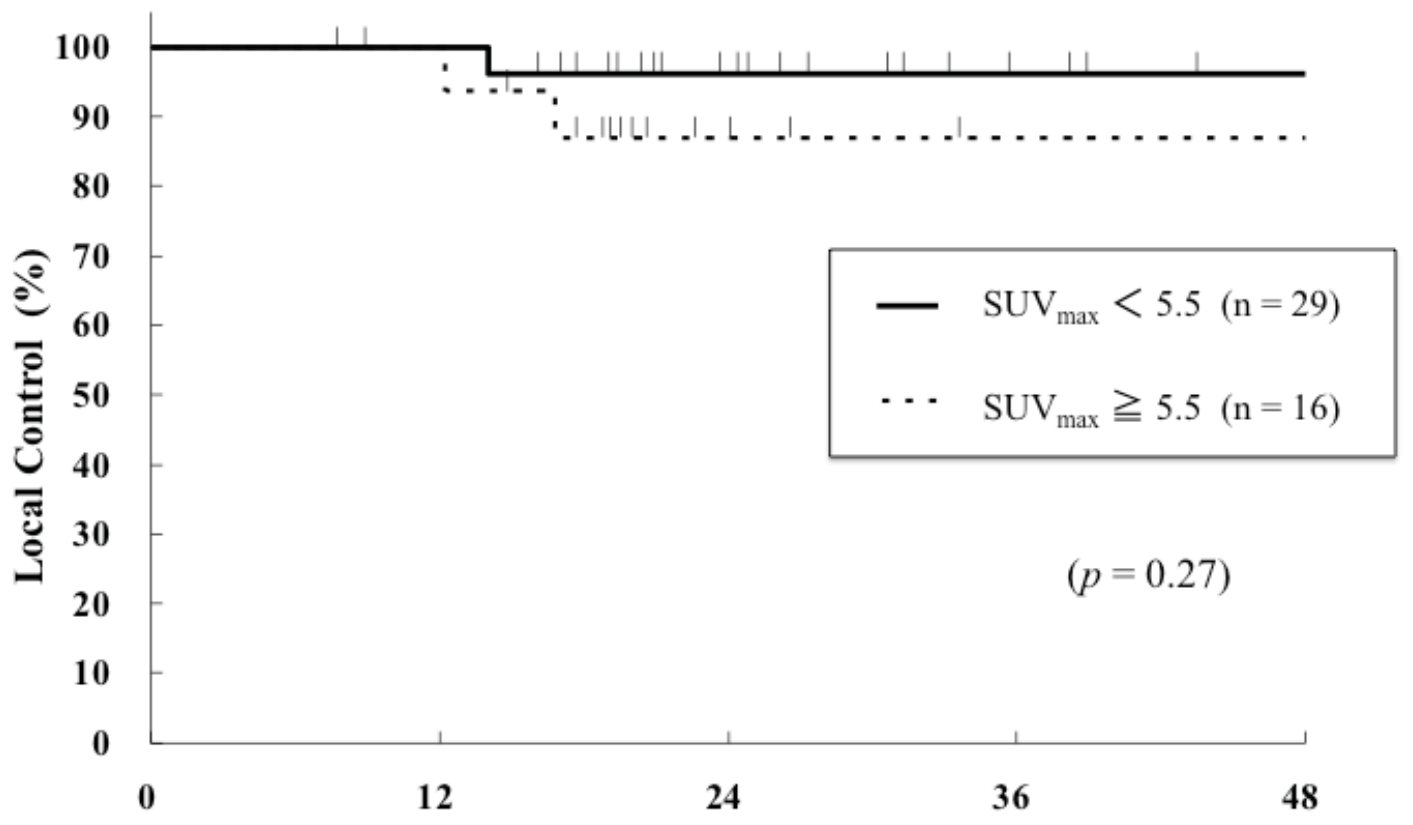

\section{Months}

Figure 2. Comparison of the local control rate according to the $\mathrm{SUV}_{\max }$ of pretreatment ${ }^{18} \mathrm{~F}$-fluorodeoxyglucose-positron emission tomography in patients with stage I non-small cell lung cancer following treatment with carbon ion radiotherapy. $\mathrm{SUV}_{\max }$, maximum standardized uptake value.

a poorer differentiation in NSCLC. van Baardwijk et al (30) revealed that high $\mathrm{SUV}_{\max }$ tumors exhibited significantly higher hypoxic marker (hypoxia-inducible factor $1-\alpha$ and glucose transporter 1) expression compared with lower $\mathrm{SUV}_{\max }$ tumors. These results suggest that a higher FDG uptake is an indicator of malignant and radioresistant characteristics, which means that these tumors may progress. As the biological and physiological advantages of $\mathrm{C}$-ion $\mathrm{RT}$ produce a high local control rate even for radioresistant tumors (for example, hypoxic cells), FDG-PET was a significant prognostic factor for PFS and OS, but not local control, in the present study.

The cut-off value for $\mathrm{SUV}_{\max }$ remains controversial. A previous study has indicated that an $\mathrm{SUV}_{\max }$ of 5-7 in tumors was important for predicting outcomes (26). However, these values depend on patient characteristics, treatments and 


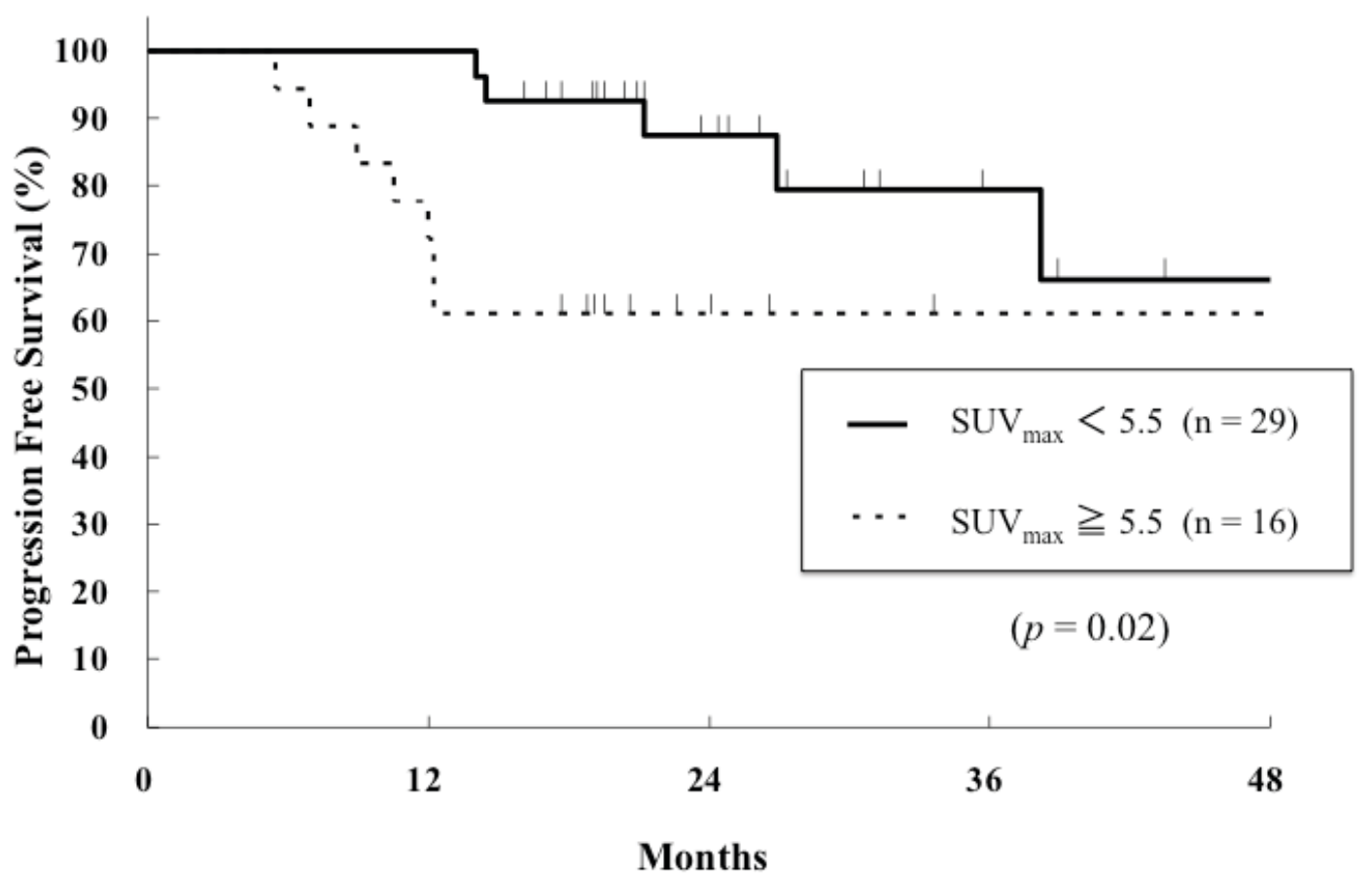

Figure 3. Progression-free survival curve according to the pretreatment SUVmax on ${ }^{18} \mathrm{~F}$-fluorodeoxyglucose-positron emission tomography in patients with stage I non-small cell lung cancer following treatment with carbon ion radiotherapy. $\mathrm{SUV}_{\max }$, maximum standardized uptake value.

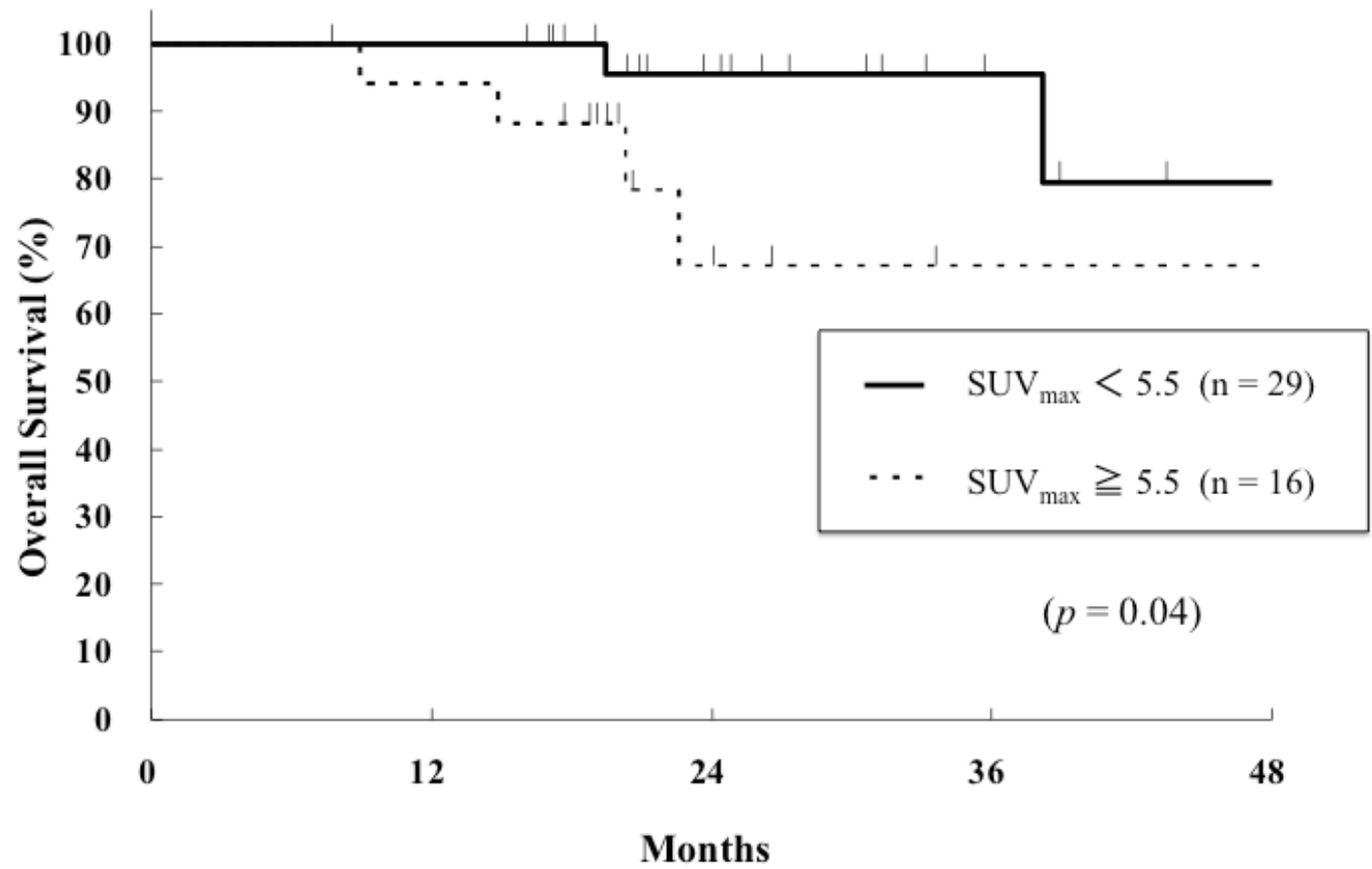

Figure 4. Overall survival curve according to the pretreatment SUVmax on ${ }^{18} \mathrm{~F}$-fluorodeoxyglucose-positron emission tomography in patients with stage I non-small cell lung cancer following treatment with carbon ion radiotherapy. $\mathrm{SUV}_{\max }$, maximum standardized uptake value.

radiation doses. Additional studies are required to validate the findings of the present study in this regard.

Although $\mathrm{SUV}_{\max }$ has been the focus of numerous studies, the usefulness of other PET parameters has also been reported. Satoh et al (31) reported that metabolic tumor volume, in addition to $\mathrm{SUV}_{\max }$, was significantly associated with outcomes following SBRT in lung cancer. Generally, the $\mathrm{SUV}_{\text {max }}$ of lung tumors is affected by the partial volume effect due to its respiratory movement, and a previous study (32) has attempted to correct this effect by using four-dimensional PET/CT. Salavati et al (32) demonstrated that semi-automated correction for the partial volume effect improved the accuracy of FDG quantification for malignant lesions of the lung.

The present study revealed that $\mathrm{C}$-ion $\mathrm{RT}$ is an effective and safe treatment for stage I NSCLC. The 2-year local control 
rate and OS were 93 and $89 \%$, respectively, and the rate of radiation pneumonitis that was above grade 2 was only $4 \%$. These results are comparable to that of a previous study of C-ion RT (8). However, additional follow-up is required to assess long-term outcomes and late adverse events following C-ion RT.

In conclusion, patients with a pretreatment $\mathrm{SUV}_{\max }$ of $\geq 5.5$ exhibited a worse 2-year PFS and OS compared with those with an $\mathrm{SUV}_{\max }$ of $<5.5$. These results indicate that pretreatment $\mathrm{SUV}_{\text {max }}$ is a prognostic marker that could be used to identify high-risk patients with NSCLC. Based on the results of the present study, patients with a higher $\mathrm{SUV}_{\max }$ may benefit from adjuvant chemotherapy following $\mathrm{C}$-ion $\mathrm{RT}$ to decrease the risk of distant metastasis. Additional studies are warranted to determine if pretreatment $\mathrm{SUV}_{\max }$ is associated with long-term prognosis.

\section{Acknowledgements}

The abstract was presented at the ASTRO 56th annual meeting Sep 14-17 2014 in San-Francisco, CA and published as abstract no. 3007 in International Journal of Radiation Oncology, Biology, Physics 90 (S607): 2014.

\section{References}

1. Nakamura K, Ukawa S, Okada E, Hirata M, Nagai A Yamagata Z, Ninomiya T, Muto K, Kiyohara Y, Matsuda K, et al: Characteristics and prognosis of Japanese male and female lung cancer patients: The BioBank Japan Project. J Epidemiol 27: S49-S57, 2017.

2. Palma D, Visser O, Lagerwaard FJ, Belderbos J, Slotman BJ and Senan S: Impact of introducing stereotactic lung radiotherapy for elderly patients with stage I non-small-cell lung cancer: A population-based time-trend analysis. J Clin Oncol 28: 5153-5159, 2010.

3. Timmerman R, Paulus R, Galvin J, Michalski J, Straube W, Bradley J, Fakiris A, Bezjak A, Videtic G, Johnstone D, et al: Stereotactic body radiation therapy for inoperable early stage lung cancer. JAMA 303: 1070-1076, 2010.

4. Onishi H, Shirato H, Nagata Y, Hiraoka M, Fujino M, Gomi K, Karasawa K, Hayakawa K, Niibe Y, Takai Y, et al: Stereotactic body radiotherapy (SBRT) for operable stage I non-small-cell lung cancer: Can SBRT be comparable to surgery? Int J Radiat Oncol Biol Phys 81: 1352-1358, 2011.

5. Kanai T, Endo M, Minohara S, Miyahara N, Koyama-ito H, Tomura H, Matsufuji N, Futami Y, Fukumura A, Hiraoka T, et al: Biophysical characteristics of HIMAC clinical irradiation system for heavy-ion radiation therapy. Int J Radiat Oncol Biol Phys 44 201-210, 1999

6. Schulz-Ertner D and Tsujii H: Particle radiation therapy using proton and heavier ion beams. J Clin Oncol 25: 953-964, 2007.

7. Ando K and Kase Y: Biological characteristics of carbon-ion therapy. Int J Radiat Biol 85: 715-728, 2009.

8. Miyamoto T, Baba M, Sugane T, Nakajima M, Yashiro T, Kagei K, Hirasawa N, Sugawara T, Yamamoto N, Koto M, et al: Carbon ion radiotherapy for stage I non-small cell lung cancer using a regimen of four fractions during 1 week. J Thorac Oncol 2: 916-926, 2007.

9. Miyamoto T, Baba M, Yamamoto N, Koto M, Sugawara T, Yashiro T, Kadono K, Ezawa H, Tsujii H, Mizoe JE, et al: Curative treatment of Stage I non-small-cell lung cancer with carbon ion beams using a hypofractionated regimen. Int J Radiat Oncol Biol Phys 67: 750-758, 2007.

10. Iwata H, Murakami M, Demizu Y, Miyawaki D, Terashima K, Niwa Y, Mima M, Akagi T, Hishikawa Y and Shibamoto Y: High-dose proton therapy and carbon-ion therapy for stage I non-small cell lung cancer. Cancer 116: 2476-2485, 2010.

11. Shirai K, Nakagawa A, Abe T, Kawahara M, Saitoh J, Ohno T and Nakano T: Use of FDG-PET in radiation treatment planning for thoracic cancers. Int J Mol Imaging 2012: 609545, 2012.
12. Lardinois D, Weder W, Hany TF, Kamel EM, Korom S, Seifert B von Schulthess GK and Steinert HC: Staging of non-small-cell lung cancer with integrated positron-emission tomography and computed tomography. N Engl J Med 348: 2500-2507, 2003.

13. Pieterman RM, Van Putten JW, Meuzelaar JJ, Mooyaart EL, Vaalburg W, Koëter GH, Fidler V, Pruim J and Groen HJ: Preoperative staging of non-small-cell lung cancer with positronemission tomography. N Engl J Med 343: 254-261, 2000.

14. Lammering G, De Ruysscher D, van Baardwijk A, Baumert BG, Borger J, Lutgens L, van den Ende P, Ollers M and Lambin P: The use of FDG-PET to target tumors by radiotherapy. Strahlenther Onkol 186: 471-481, 2010.

15. Dooms C, Verbeken E, Stroobants S, Nackaerts K, De Leyn P and Vansteenkiste J: Prognostic stratification of stage IIIA-N2 non-small-cell lung cancer after induction chemotherapy: A model based on the combination of morphometric-pathologic response in mediastinal nodes and primary tumor response on serial 18-fluoro-2-deoxy-glucose positron emission tomography. J Clin Oncol 26: 1128-1134, 2008.

16. Mac Manus MP, Hicks RJ, Matthews JP, Wirth A, Rischin D and Ball DL: Metabolic (FDG-PET) response after radical radiotherapy/chemoradiotherapy for non-small cell lung cancer correlates with patterns of failure. Lung Cancer 49: 95-108, 2005.

17. Huang W, Zhou T, Ma L, Sun H, Gong H, Wang J, Yu J and Li B: Standard uptake value and metabolic tumor volume of ${ }^{18} \mathrm{~F}$-FDG PET/CT predict short-term outcome early in the course of chemoradiotherapy in advanced non-small cell lung cancer. Eur J Nucl Med Mol Imaging 38: 1628-1635, 2011.

18. Berghmans T, Dusart M, Paesmans M, Hossein-Foucher C, Buvat I, Castaigne C, Scherpereel A, Mascaux C, Moreau M, Roelandts M, et al: Primary tumor standardized uptake value (SUVmax) measured on fluorodeoxyglucose positron emission tomography (FDG-PET) is of prognostic value for survival in non-small cell lung cancer (NSCLC): A systematic review and meta-analysis (MA) by the European Lung Cancer Working Party for the IASLC Lung Cancer Staging Project. J Thorac Oncol 3: 6-12, 2008.

19. Tashiro M, Ishii T, Koya J, Okada R, Kurosawa Y, Arai K, Abe S, Ohashi Y, Shimada H, Yusa K, et al: Technical approach to individualized respiratory-gated carbon-ion therapy for mobile organs. Radiol Phys Technol 6: 356-366, 2013.

20. National Cancer Institute: Common Terminology Criteria for Adverse Events (CTCAE v4.0). https://ctep.cancer.gov/protocolDevelopment/electronic_applications/ctc.html. Updated November 14, 2016.

21. Coon D, Gokhale AS, Burton SA, Heron DE, Ozhasoglu C and Christie N: Fractionated stereotactic body radiation therapy in the treatment of primary, recurrent, and metastatic lung tumors: The role of positron emission tomography/computed tomography-based treatment planning. Clin Lung Cancer 9: 217-221, 2008

22. Burdick MJ, Stephans KL, Reddy CA, Djemil T, Srinivas SM and Videtic GM: Maximum standardized uptake value from staging FDG-PET/CT does not predict treatment outcome for early-stage non-small-cell lung cancer treated with stereotactic body radiotherapy. Int J Radiat Oncol Biol Phys 78: 1033-1039, 2010.

23. Takeda A, Yokosuka N, Ohashi T, Kunieda E, Fujii H, Aoki Y, Sanuki N, Koike N and Ozawa Y: The maximum standardized uptake value (SUVmax) on FDG-PET is a strong predictor of local recurrence for localized non-small-cell lung cancer after stereotactic body radiotherapy (SBRT). Radiother Oncol 101: 291-297, 2011.

24. Chang JY, Liu H, Balter P, Komaki R, LiaoZ, Welsh J, Mehran RJ, Roth JA and Swisher SG: Clinical outcome and predictors of survival and pneumonitis after stereotactic ablative radiotherapy for stage I non-small cell lung cancer. Radiat Oncol 7: 152, 2012.

25. Clarke K, Taremi M, Dahele M, Freeman M, Fung S, Franks K, Bezjak A, Brade A, Cho J, Hope A, et al: Stereotactic body radiotherapy (SBRT) for non-small cell lung cancer (NSCLC): Is FDG-PET a predictor of outcome? Radiother Oncol 104: 62-66, 2012.

26. Na F, Wang J, Li C, Deng L, Xue J and Lu Y: Primary tumor standardized uptake value measured on F18-Fluorodeoxyglucose positron emission tomography is of prediction value for survival and local control in non-small-cell lung cancer receiving radiotherapy: Meta-analysis. J Thorac Oncol 9: 834-842, 2014.

27. Goodgame B, Pillot GA, Yang Z, Shriki J, Meyers BF, Zoole J, Gao F, Dehdashti F, Patterson A, Siegel BA and Govindan R: Prognostic value of preoperative positron emission tomography in resected stage I non-small cell lung cancer. J Thorac Oncol 3: $130-134,2008$. 
28. Nair VS, Krupitskaya Y and Gould MK: Positron emission tomography $18 \mathrm{~F}$-fluorodeoxyglucose uptake and prognosis in patients with surgically treated, stage I non-small cell lung cancer: A systematic review. J Thorac Oncol 4: 1473-1479, 2009.

29. Vesselle H, Schmidt RA, Pugsley JM, Li M, Kohlmyer SG, Vallires E and Wood DE: Lung cancer proliferation correlates with [F-18]fluorodeoxyglucose uptake by positron emission tomography. Clin Cancer Res 6: 3837-3844, 2000.

30. van Baardwijk A, Dooms C, van Suylen RJ, Verbeken E, Hochstenbag M, Dehing-Oberije C, Rupa D, Pastorekova S, Stroobants S, Buell U, et al: The maximum uptake of (18) F-deoxyglucose on positron emission tomography scan correlates with survival, hypoxia inducible factor-lalpha and GLUT-1 in non-small cell lung cancer. Eur J Cancer 43: 1392-1398, 2007.
31. Satoh Y, Onishi H, Nambu A and Araki T: Volume-based parameters measured by using FDG PET/CT in patients with stage I NSCLC treated with stereotactic body radiation therapy: Prognostic value. Radiology 270: 275-281, 2014.

32. Salavati A, Borofsky S, Boon-Keng TK, Houshmand S, Khiewvan B, Saboury B, Codreanu I, Torigian DA, Zaidi H and Alavi A: Application of partial volume effect correction and 4D PET in the quantification of FDG avid lung lesions. Mol Imaging Biol 17: 140-148, 2015. 